\title{
Role of immunohistochemistry in diagnosis of nasopharyngeal tumours
}

\author{
J BOSQ,${ }^{*}$ KC GATTER, ${ }^{*}$ C MICHEAU, $†$ DY MASON* \\ From the *Nuffield Department of Pathology, John Radcliffe Hospital, Headington, Oxford, and the \\ $\dagger$ Departement d'Anatomie Pathologique, Histopathologie "A", Institut Gustave Roussy, 94800 Villejuif, \\ France
}

SUMMARY To evaluate the usefulness of immunocytochemistry in the differential diagnosis of nasopharyngeal tumours, 35 undifferentiated nasal carcinomas were examined with a panel of monoclonal antibodies against a wide variety of epithelial and non-epithelial antigens. The results were compared with those obtained from a series of nasopharyngeal tumours comprising three squamous cell carcinomas, six lymphomas, one rhabdomyosarcoma, and one yolk sac tumour. All of the carcinomas were positive with at least one of the antiepithelial markers and negative for the leucocyte common antigen and were clearly distinguishable from the nasopharyngeal lymphomas, which gave the opposite staining pattern. It was concluded that such a panel of monoclonal antibodies would be extremely useful for the differential diagnosis of nasopharyngeal tumours, particularly with small or technically inadequate biopsies.

Most malignancies of the head and neck are easily recognisable as squamous cell carcinomas and, therefore, present few problems for histological diagnosis. The exception is malignancy of the nasopharynx, where many of the tumours are undifferentiated carcinomas that can be difficult to distinguish from other neoplasms such as malignant lymphoma. Although these tumours are rare in most of Europe and the United States, they are relatively common in north Africa, South East Asia, and southern China.

The present study was undertaken to establish the value of a panel of monoclonal antibodies in the diagnosis of so called undifferentiated carcinomas of nasopharyngeal type. A series of routinely processed nasopharyngeal carcinomas was compared with a small number of other nasopharyngeal tumours of both epithelial and non-epithelial type with which they might be confused morphologically.

\section{Material and methods}

TISSUE SAMPLES

Forty six nasopharyngeal biopsies were obtained from the files of the histopathology departments of the Institut Gustave Roussy and the John Radcliffe Hospital. All the biopsies had been routinely pro-

Accepted for publication 10 April 1985 cessed by formalin fixation and paraffin embedding. The biopsies were classified as follows: thirty five undifferentiated carcinomas of nasopharyngeal type; three squamous cell carcinomas (two well and one poorly differentiated); six non-Hodgkin's lymphomas (three lymphoblastic, one centroblastic, and two immunoblastic); one rhabdomyosarcoma; one metastasis from a yolk sac tumour.

IMMUNOHISTOLOGICAL STAINING

Sections were immunostained either by an immunoperoxidase procedure in three stages or by the immunoalkaline phosphatase antialkaline phosphatase procedure. ${ }^{12}$

IMMUNOHISTOCHEMICAL REAGENTS

Table 1 shows the details of the monoclonal antibodies used in this study. Peroxidase conjugated swine antirabbit immunoglobulin and peroxidase conjugated rabbit antimouse immunoglobulin were obtained from Dakopatts A/S. Alkaline phosphatase antialkaline phosphatase immune complexes were prepared according to the method described by Cordell et al. ${ }^{2}$ Diaminobenzidine tetrahydrochloride, naphthol-AS-MX phosphate, and fast red were obtained from the Sigma Chemical Company. Tris buffered saline was prepared by adding a tenth volume of $0.5 \mathrm{M}$ Tris hydrochloric acid buffer $(\mathrm{pH}$ $7 \cdot 6$ ) to $0 \cdot 15 \mathrm{M}$ saline. 
Table 1 Panel of monoclonal antibodies used in this study

\begin{tabular}{|c|c|c|}
\hline Antibody & $\begin{array}{l}\text { Source and } \\
\text { reference }\end{array}$ & Antigen \\
\hline $\begin{array}{l}\text { KLI } \\
\text { CAM 5.2 }\end{array}$ & $\left.\begin{array}{l}3 \\
4\end{array}\right\}$ & Cytokeratins \\
\hline E29 & 1,5 & $\begin{array}{l}\text { Epithelial membrane antigen } \\
\text { (Human milk fat globule membrane) }\end{array}$ \\
\hline 11.285 .14 & 5 & Carcinoembryonic antigen \\
\hline $\begin{array}{l}\mathrm{PD} 7 / 26 \\
2 \mathrm{~B} 11\end{array}$ & $\left.\begin{array}{l}7 \\
7\end{array}\right\}$ & Leucocyte common antigen \\
\hline $\begin{array}{l}\text { S1-61 } \\
\text { S3-77 }\end{array}$ & & S100 protein \\
\hline NK1/C3 & 9 & Melanoma associated antigen \\
\hline
\end{tabular}

\section{Results}

Table 2 summarises the results obtained in this study from a series of 46 nasopharyngeal tumours.

\section{UNDIFFERENTIATED CARCINOMAS OF \\ NASOPHAR YNGEAL TYPE}

All 35 cases showed some degree of positivity with the anticytokeratin KL1 (Fig 1a), whereas two cases were completely negative with CAM 5.2. In general KL1 gave stronger and more extensive staining than CAM 5.2 and was particularly useful for highlighting small islands of carcinoma against a dense lymphoid background (Fig. 1b). Thirty one of the tumours expressed weak focal staining for epithelial membrane antigen with only a few positive cells in some cases. The anti-S100 protein antibodies gave weak labelling in about half of the cases of undifferentiated carcinomas of nasopharyngeal type. All 35 cases were completely negative for the antileucocyte common antigen (Fig. 1c) and for the antimelanoma associated antigen recognised by antibody NK1/C3.

\section{SQUAMOUS CELL CARCINOMAS}

All three squamous cell carcinomas were positively labelled with both of the anticytokeratin antibodies, with KL1 generally showing a stronger intensity of staining than CAM 5.2. There was no correlation between the degree of differentiation and the intensity of the labelling. Epithelial membrane antigen and carcinoembryonic antigen were focally distributed with carcinoembryonic antigen being restricted to the well differentiated areas. Scattered tumour cells also showed some positivity with both antibodies against the $\mathrm{S} 100$ protein. All three tumours were negative for the leucocyte common antigen and for the melanoma associated antigen.

\section{LYMPHOMAS}

In contrast to the carcinoma, all six lymphomas were negative for the cytokeratins, epithelial membrane antigen, and carcinoembryonic antigen. Five lymphomas showed the characteristic staining of the surface membrane for the leucocyte common antigen, whereas the sixth, an immunoblastic lymphoma, although negative for this antigen, was confirmed as a lymphoma by the presence of immunoglobulin light chain restriction. One of the two immunoblastic lymphomas was weakly positive for the S100 protein, although all were negative for the melanoma associated antigen recognised by antibody NK1-C3.

\section{RHABDOMYOSARCOMA}

This tumour showed weak intracytoplasmic positivity for the S100 protein but was negative with all of the other markers in this study.

\section{YOLK SAC TUMOUR METASTASIS}

This tumour was, morphologically, a characteristic yolk sac tumour, which was confirmed by its strong positivity for $\alpha$-fetoprotein. It was positively labelled by both the anticytokeratin antibodies and weakly positive for epithelial membrane antigen, S100 protein, and the antimelanoma associated antigen.

Table 2 Results of staining with panel of monoclonal antibodies

\begin{tabular}{|c|c|c|c|c|c|c|c|c|c|c|}
\hline & $K L I$ & $C A M 5 \cdot 2$ & $E 29$ & $C E A$ & $2 B 11$ & $P D 7 / 26$ & $S 1-61$ & S3-77 & $N K 1-C 3$ & $\alpha$-fetoprotein \\
\hline $\begin{array}{l}\text { Squamous cell carcinoma } \\
(n=3) \\
\text { Undifferentiated } \\
\text { carcinoma of }\end{array}$ & + & + & + & $+\underset{(2 / 3)}{+}$ & - & - & + & + & - & not done \\
\hline $\begin{array}{l}\text { nasopharyngeal type } \\
(n=35)\end{array}$ & + & $\begin{array}{l}+ \\
(33 / 35)\end{array}$ & $\begin{array}{l}+ \\
(31 / 35)\end{array}$ & - & - & - & $+\underset{(14 / 35)}{+}$ & $\begin{array}{l}+ \\
(19 / 35)\end{array}$ & - & not done \\
\hline $\begin{array}{l}\text { Lymphoma } \\
(\mathrm{n}=6)\end{array}$ & - & - & - & - & $\begin{array}{l}+ \\
(4 / 6)\end{array}$ & $\stackrel{+}{(5 / 6)}$ & - & $(5 / 6)$ & - & not done \\
\hline $\begin{array}{l}\text { Rhabdomyosarcoma } \\
(n=1) \\
\text { Yolk sac tumour }\end{array}$ & - & - & - & - & - & - & - & + & - & not done \\
\hline $\begin{array}{l}\text { (metastasis) } \\
(n=1)\end{array}$ & + & + & + & - & - & - & + & + & + & + \\
\hline
\end{tabular}

Unless otherwise stated (by proportions in parentheses) $+=$ all cases positive, $-=$ all cases negative. 


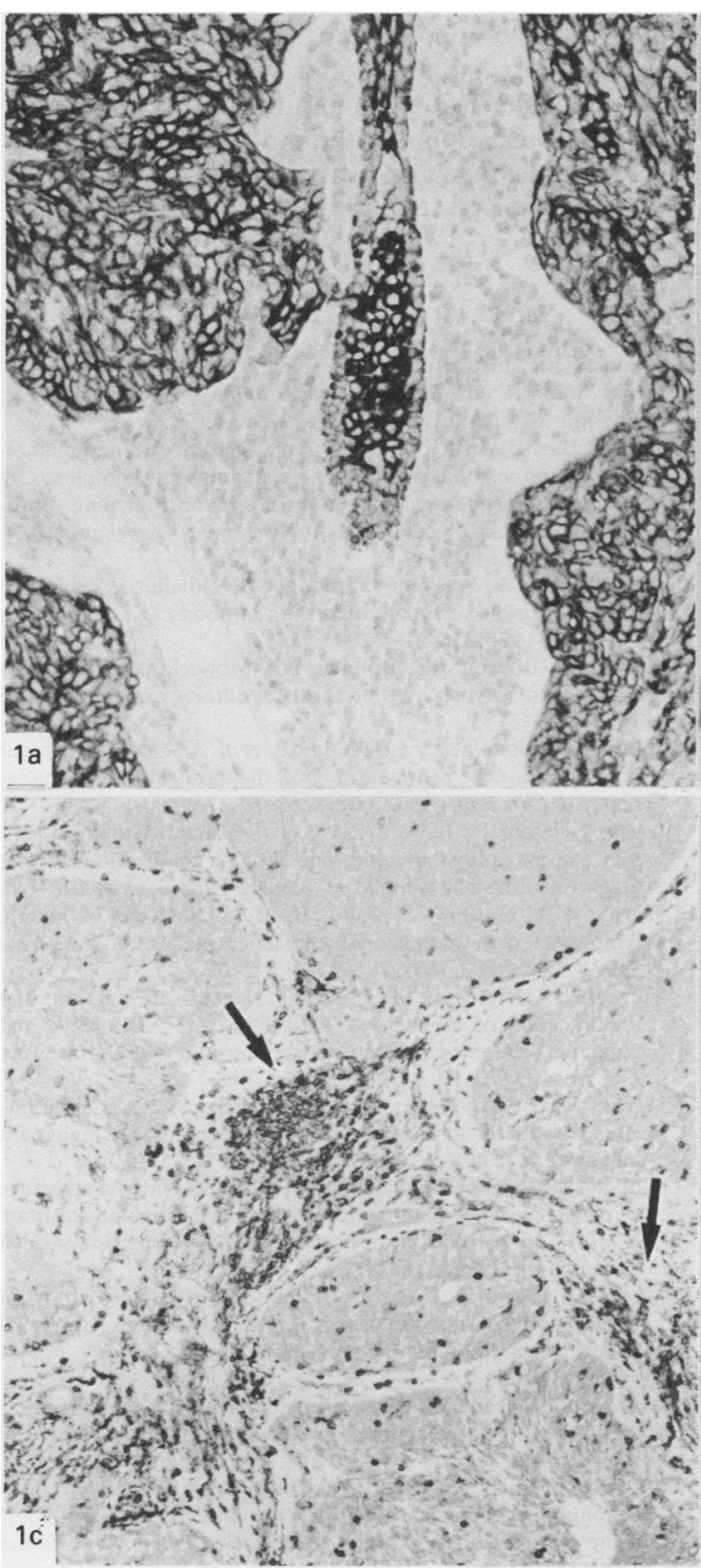

\section{Discussion}

The present study confirms immunohistologically the accepted view that the entity of undifferentiated carcinomas of nasopharyngeal type represents a single tumour type of epithelial origin. ${ }^{10-13}$ The morphological recognition of these undifferentiated carcinomas, however, generally requires consider-

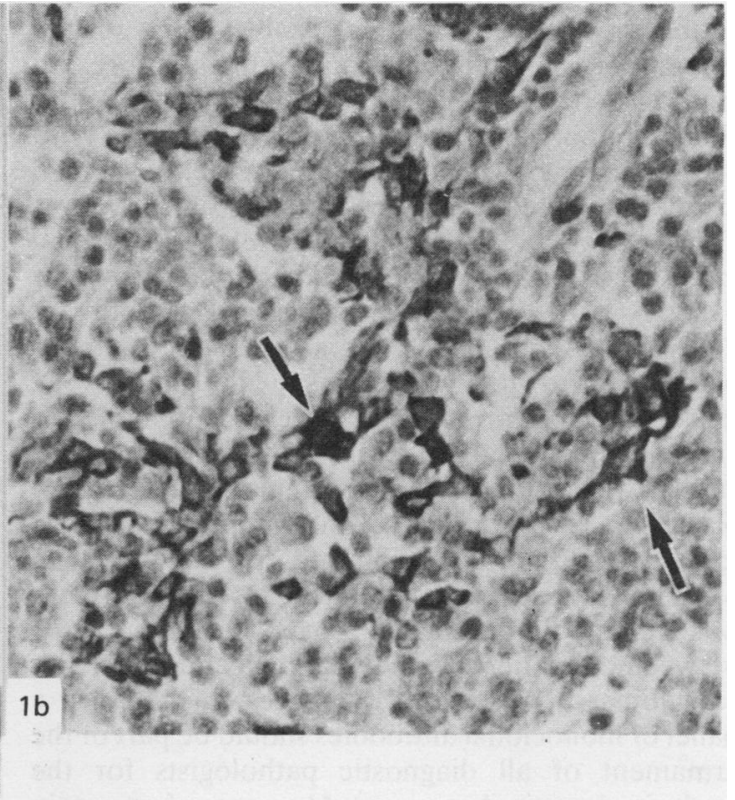

Fig 1a Undifferentiated carcinoma of nasopharyngeal type showing homogeneous staining of tumour with anticytokeratin KL1. Surrounding lymphoid cells are completely unstained. (Alkaline phosphatase. $\times 250$.)

Fig 1b Undifferentiated carcinomas of nasopharyngeal type of diffuse type showing separated tumour cells (arrows) clearly highlighted by anticytokeratin KL1 from unstained lymphoid background.

(Immunoperoxidase. $\times 500$.)

Fig 1c Sections of undifferentiated carcinomas of nasopharyngeal type stained for leucocyte common antigen, showing unstained epithelial tumour contrasting with positive lymphoid infiltrate (arrows).

able experience of these tumours, and their differentiation from lymphoma and sarcoma continues to cause difficulty in diagnostic practice. Preliminary studies have indicated that immunohistological examination might be of assistance in their differential diagnosis. 112 These studies, however, relied mainly on the use of polyclonal antisera, with their inherent drawbacks compared with monoclonal 
antibodies, and contained no reliable agents for the recognition of lymphomas in routinely processed material. We used several recently produced monoclonal antibodies against both epithelial ${ }^{34}$ and lymphoid antigens, ${ }^{7}$ which are relatively well preserved in routinely fixed and processed material.

The clinical usefulness of this panel of monoclonal antibodies has been established in our laboratory on a series of 120 routinely processed tumours of uncertain origin, which were referred by other pathologists owing to diagnostic difficulties. ${ }^{14}$ Of these 120 tumours, 105 could be clearly identified as either lymphomas (79 cases) or carcinomas (28 cases), with four melanoma/seminoma and neuroblastoma. This left only seven tumours whose true identity could not be established. Clinical follow up of these patients showed that at three years there was a marked difference in prognosis of about $25 \%$ between the patients with lymphoma and those with carcinoma. It was therefore suggested that such a panel of monoclonal antibodies should be part of the armament of all diagnostic pathologists for the analysis of routinely processed tumours of uncertain origin.

This study has shown that a large series of undifferentiated nasopharyngeal carcinomas can be reliably distinguished from other non-epithelial tumours of the nasopharynx, including malignant lymphomas. The use of a panel of monoclonal antibodies is especially valuable as most types of tumour can be recognised by positive staining as well as the absence of markers associated with other types. Although epithelial membrane antigen was a reliable epithelial marker in this study, its presence has been shown in a few lymphoid tumours, ${ }^{6}$ and it therefore cannot be relied on alone as an epithelial specific marker.

These findings are particularly relevant in practice as nasopharyngeal biopsies are often small and considerably distorted by crush artefact. That these monoclonal antibodies work reliably in routinely processed materials is a further considerable asset as the malignant nature of nasopharyngeal biopsies is often not appreciated until the material has been examined after fixation and paraffin embedding. This means that the diagnostic dilemma as to whether a nasopharyngeal tumour is a carcinoma or a lymphoma, with its consequent effect on the treatment and prognosis of the patient, can now be reliably solved by any pathologist augmenting the routine histological examination with immunocytochemistry using such a panel of monoclonal antibodies.
This work was supported by the Association pour la Recherche sur le Cancer, the Wellcome Trust, and the Leukaemia Research Fund. We thank all those who generously donated the monoclonal antibodies used and Lesley Watts for typing the manuscript.

\section{References}

' Gatter KC, Falini B, Mason DY. The use of monoclonal antibodies in histopathological diagnosis. In: Anthony $\mathbf{P}$, MacSween R, eds. Recent advances in histopathology. Vol 12. London: Churchill Livingstone, 1984:35-67.

${ }^{2}$ Cordell JL, Falini B, Erber WN, et al. Immunoenzymatic labelling of monoclonal antibodies using immune complexes of alkaline phosphatase and monoclonal anti-alkaline phosphatase (APAAP complexes). Histochem Cytochem 1984; 32:219-29.

${ }^{3}$ Viac J, Reano A, Brochier J, Staquet MJ, Thivolet J. Reactivity pattern of a monoclonal antikeratin antibody (KL1). J Invest Dermatol 1983;81:351-54.

${ }^{4}$ Makin CA, Bobrow LG, Bodmer WF. Monoclonal antibody to cytokeratin for use in routine histopathology. J Clin Pathol 1984;37:975-83.

s Gatter KC, Abdulaziz Z, Beverley P, et al. Use of monoclonal antibodies for the histopathological diagnosis of human malignancy. J Clin Pathol 1982;35: 1253-67.

- Delsol G, Stein H, Pulford KAF, et al. Human lymphoid cells may express epithelial membrane antigen. Implications for the diagnosis of human neoplasms. Lancet 1984:ii;1124-9.

' Warnke RA, Gatter KC, Falini B, et al. Diagnosis of human lymphoma with monoclonal anti-leucocyte antibodies. New Engl J Med 1983;309:1275-81.

8 Vanstapel MJ, Peeters B, Cordell J, et al. Production and identification of monoclonal antibodies directed against and antigenic determinent common to the $\alpha$ and $\beta$ chain of S100. Lab Invest 1985;52:232-8.

' Mackie RM, Campbell I, Turbitt ML. Use of NK1C3 monoclonal antibody in the assessment of benign and malignant melanocytic lesions. J Clin Pathol 1984;37:367-72.

${ }^{10}$ Svoboda D, Kirchner F, Shanmugaratnam K. The fine structure of nasopharyngeal carcinoma. In: Muir CS, Shanmugaratnam $\mathrm{K}$, eds. Cancer of the nasopharynx (VICC Monograph series.) Copenhagen: Munksgaard, 1967:163-71.

"Madri JA, Barwick KW. An immunohistochemical study of nasopharyngeal neoplasms using keratin antibodies. Epithelial versus non-epithelial neoplasms. Am J Surg Pathol 1982;6:143-9.

${ }^{12}$ Gusterson BA, Mitchell DP, Warburton HJ, Carter RL. Epithelial markers in the diagnosis of nasopharyngeal carcinoma: an immunocytochemical study.J Clin Pathol 1983;36:628-31.

${ }^{13}$ Shi Sr, Goodman ML, Bhan AK, Pilch BZ, Chen LB, Sun TT. Immunohistochemical study of nasopharyngeal carcinoma using monoclonal keratin antibodies. Am J Pathol 1984; 117: 53-63.

${ }^{14}$ Gatter KC, Alcock C, Heryet A, Mason DY. The clinical importance of analysing tumours of uncertain origin by immunohistological techniques. Lancet (in press).

Requests for reprints to: KC Gatter, Nuffield Department of Pathology, John Radcliffe Hospital, Headington, Oxford OX3 9DU. 